EPJ Web of Conferences 64, 06006 (2014)

DOI: $10.1051 /$ epjconf/ 20146406006

(C) Owned by the authors, published by EDP Sciences, 2014

\title{
The reflection component in NS LMXBs
}

\author{
A. D’Aí ${ }^{1, a}$, A. Papitto ${ }^{2}$, T. Di Salvo ${ }^{1}$, R. laria ${ }^{1}$, N.R. Robba ${ }^{1}$, E. Egron ${ }^{3}$, and S. Piraino ${ }^{4}$ \\ ${ }^{1}$ Dipartimento di Fisica e Chimica, via Archirafi 36, 90123 Palermo, Italy \\ ${ }^{2}$ ICE, IEEC-CSIC, Barcelona, Spain \\ ${ }^{3}$ INAF Osservatorio Astronomico di Cagliari, Italy \\ ${ }^{4}$ INAF-IASF di Palermo, Italy
}

\begin{abstract}
Thanks to the good spectral resolution and large effective area of the EPIC/PN instrument on board of XMM-Newton, we have at hand a large number of observations of accreting low-mass X-ray binaries, that allow for the fist time a comprehensive view on the characteristics of the reflection component at different accretion regimes and to probe the effects of a magnetosphere on its formation. We focus here on a comparative analysis of the reflection component from a series of spectroscopic studies on selected sources: $4 \mathrm{U}$ 1705-44, observed both in the soft and hard state, the pulsating ms pulsars SAX J1808.43658 and IGR J17511-3057, and the intermittent pulsar HETE J1900-2455. Although the sources can present very similar accretion rates and continuum shapes, the reflection parameters do not generally result the same, moreover the effect of a magnetosphere on the formation of the reflection component appears elusive.
\end{abstract}

\section{Introduction}

Broad iron emission lines have been observed in many low-mass X-ray binaries (LMXBs) hosting both neutron stars (NSs) and black-holes with high statistics and good spectral resolution by the EPIC/PN on board of XMM-Newton. There is growing evidence that these lines are formed by reflection of corona/boundary layer photons in the accretion disk and broadened by a combination of dynamical and relativistic effects. Diagnostics from the line shapes and broadband reflection continuum may provide constraints on the dimensions and the physical characteristics of the boundary layer, or of the magnetospheric boundary, if the NS owes a magnetic field able to drive the accretion process. Comparison of the reflection component in pulsating and non-pulsating NS, or in different accretion states may thus provide an important mean to study the accretion flow geometry and physics close to the NS.

NSs accreting through Roche-lobe overflow can either be found in a soft, or in a hard spectral state. The soft state is characterized by high accretion rates (luminosity $\geq 0.1 \mathrm{~L}_{E d d}$ ), total flux mostly in the 1-10 keV range, spectrum composed of a soft $\sim 1 \mathrm{keV}$ thermal disk emission and optically thick boundary layer emission. The hard state is, on the contrary, characterized by lower accretion rates, relatively small, or undetected, disk emission and a dominating hard, optically thin, Comptonized component extending well above $10 \mathrm{keV}$. In both states, a reflection component is present and appears relativistically broadened.

\footnotetext{
a e-mail: antonino.dai@unipa.it
}

This is an Open Access article distributed under the terms of the Creative Commons Attribution License 2.0, which permits unrestricted use, distribution, and reproduction in any medium, provided the original work is properly cited. 

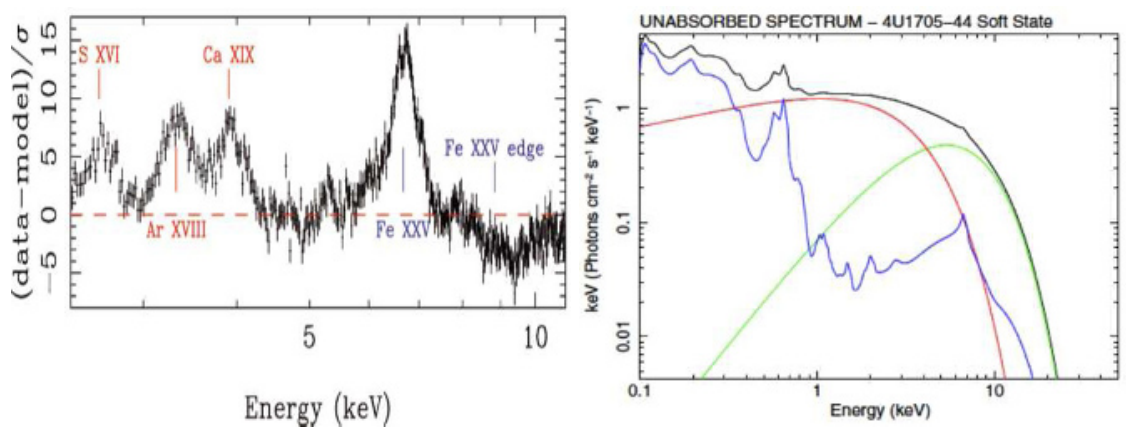

Figure 1. Residual pattern in the EPIC/PN spectrum of continuum emission without the reflection component [see 2], where emission from resonant S XVI, Ar XVIII, Ca XIX, Fe XXV and an absorption edge of Fe XXV are clearly detected. Unabsorbed fluxes from the best-fitting models of the XMM observation of the atoll source 4U 1705-44 in the soft state. Disk emission in red, boundary layer emission in green, reflection component (with a relativistic smearing) in blue [see 5].

\section{The reflection component in hard and soft states in non-pulsating NSs}

To study the change on the reflected spectrum in the two spectral states, we used two long XMM observations of the bright atoll source $4 \mathrm{U} 1705-44$, which can be considered the prototype of accreting NS LMXB swinging between hard and soft states. In the soft state, reflection is at best constrained, given the usually high signal-to-noise ratio in the Fe $\mathrm{K} \alpha$ region [see also 1-4]. Using a self-consistent reflection model ( $r e f b b)$ that adopts a black-body incident spectrum as source of irradiation, convolved with a relativistic smearing kernel (rdblur), we found that the temperature of the incident flux and the temperature of the harder continuum X-ray component do well agree, leading to the conclusion that the boundary layer emission is the source of the disk reflected component $[5,6]$. The ionization state of the reflecting matter is constrained $2.2<\log \xi<2.7$, a value that sets the iron He-like line as the most prominent feature. In this state we found converging evidence that the boundary layer outer radius, as derived by the inner disk radius of the reflection component, is $\sim 2$ times the NS radius, thus being not only optically thick but also geometrically thick (see the spectral decomposition for this state in the right panel of Fig.1). Reflection is most clearly observed and determined by the shape of the iron fluorescence line, but other He-like resonant lines of lighter elements could also be detected (see left panel of Fig.1). In this state, given that the incident spectrum is black-body like, no Compton hump is expected.

In the hard state, the continuum emission is dominated by an optically thin Comptonized component, with a bolometric luminosity of $3 \pm 1 \times 10^{36} \mathrm{erg} \mathrm{s}^{-1}$. A broad Fe K $\alpha$ feature at $\sim 6.4 \mathrm{keV}$, corresponding to a lower ionization state $(\log \xi<1.04)$, is present; the line has a symmetric shape and strong smearing is not detected, possibly due to an accretion disk truncated at greater distance $\left(R_{\text {in }} \sim 30 R_{g}\right)$.

\section{The accreting ms pulsars}

In ms X-ray pulsars the boundary layer size and geometry is determined by the intensity of the NS magnetic field, as it possibly truncates the accretion disk and funnels the accretion flow into the magnetic caps. In particular, for X-ray pulsars, the inner disc radius, $R_{i n}$, must be larger than the NS surface and not much larger than the co-rotation radius, defined as the point where the magnetosphere 

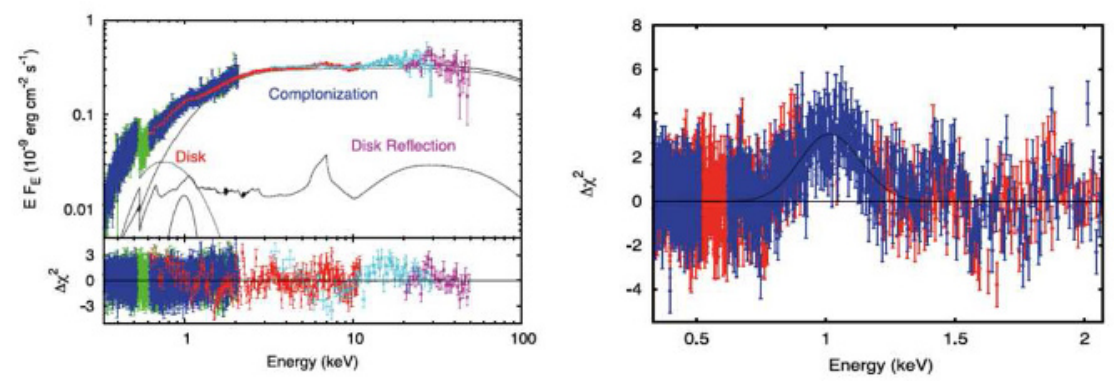

Figure 2. The broadband spectral decomposition for the intermittent pulsar HETE J1900.1 (left panel) and a blow-up of residuals around $1 \mathrm{keV}$ showing a local soft excess [see 9].

rotation equals that of an assumed Keplerian disc, $R_{c o r}=\left(G M P^{2} / 4 \pi^{2}\right)^{1 / 3}$, otherwise matter would be expelled by the centrifugal barrier.

During the 2008 outburst of SAX J1808-3658, XMM observed this source for $63 \mathrm{ks}$. The source was found in the hard state, accreting at a luminosity of $6.6 \times 10^{36} \mathrm{erg} \mathrm{s}^{-1}$. A broad (FWHM $\sim 1 \mathrm{keV}$ ) iron line was present in the spectrum. The line energy is compatible with iron in low ionization state. Interpreting the broadness as due to relativistic smearing, we found a best-fitting estimate $R_{\text {in }}=8.7$ gravitational radii, $R_{g}$, with an upper limit at $12.4 R_{g}$ corresponding to $25.6 \mathrm{~km}$ for a canonical $1.4 \mathrm{M}_{\odot}$ NS. X-ray pulsations at $401 \mathrm{~Hz}$ resulted in a co-rotational radius of $31 \mathrm{~km} \mathrm{[7].}$

In Sept. 2009 XMM observed the $244.8 \mathrm{~Hz}$ ms pulsar IGR J17511-3057 [8]. The spectral state was hard, with a luminosity of $\sim 10 \times 10^{36} \mathrm{erg} \mathrm{s}^{-1}$ (for a distance of $8 \mathrm{kpc}$ ). A reflection component was detected thanks to a broad iron fluorescence line and a Compton hump at energies above $10 \mathrm{keV}$. The disk was highly ionized, $\log (\xi)=3.0_{-0.2}^{+0.4}$, with an inner disk radius at $R_{i n}=27_{-9}^{+6} R_{g}\left(R_{\text {cor }} \sim 21 \mathrm{R}_{g}\right)$. A number of ms X-ray pulsars show also intermittent pulsations, where either the magnetic field is buried by a prolonged accretion phase or are too weak to drive a significant fraction of the accreted matter towards the magnetic caps. HETE J1900.1-2455 was found to show such behaviour, where pulsations at $377.3 \mathrm{~Hz}$ are not always detected. XMM observed this source in Sept. 2011 for 63 ks [9]. Simultaneous observation with Rossi-XTE allowed determination of the broad band X-ray emission, with an overall luminosity of $3.6 \times 10^{36} \mathrm{erg} \mathrm{s}^{-2}$. Pulsations were not detected, with an upper limit of $0.4 \%$ on the pulsed fraction. The broadband spectrum is dominated by an optically thin Comptonization with some softer continuum contribution from the disk and the magnetic cap region (Fig.2). Using self-consistent reflection models, the ionisation parameter was constrained in the range $2.8<\log (\xi)<3.0$. The inner disk radius is constrained in the $14-36 R_{g}$ range. A broad emission feature at $1 \mathrm{keV}$ (see right panel of Fig.2) was interpreted as a blending of intermediate Fe L-lines and $\mathrm{Ne}$ IX-X, however the observed flux implies significant over-abundance of these elements.

\section{Discussion}

We gave a quick summary on the spectral results of the above-mentioned, showing that the reflection component may assume widely different parameters. The soft states are usually associated with a highly ionized reflecting skin and a disk truncation due to the geometric inflation of the boundary layer $\left(10 R_{g}<R_{\text {in }}<15 R_{g}\right.$ ). We presently have not any observation of a ms X-ray pulsar in a soft state. Atoll sources enter the hard state when the luminosity drops below a certain threshold (usually a few percent of the Eddington limit), and the accretion flow close to the NS becomes optically thin. 
The hard state between pulsating and no-pulsating NS appears very similar, but in accreting ms pulsars there is evidence of an additional softer component, probably formed in the magnetic cap. 4U 1705-44 and SAX J1808.4 show a neutral/mildly ionized Fe K $\alpha$ iron line, but the relative broadening of the line is different (for a Gaussian fit to the line, the $\sigma$ is $0.41 \pm 0.08 \mathrm{keV}$ for $4 \mathrm{U} 1705-44$ and $1.1 \pm 0.2 \mathrm{keV}$ for SAX J1808), possibly indicating that the hard state in atoll sources has a larger truncation radius. In SAX J1808.4 the magnetic field drives part of the accretion flow, as pulsations are clearly detected but the magnetic field lines uplift matter extremely close to the NS, a situation that does not allow to separate the effects of a magnetosphere from a scenario of disk evaporation. SAX J1808.4 with its extreme broadness has in the sample the lowest upper limit on the inner radius, and a lower limit compatible with no disk-truncation.

In HETE J1900.1 and in IGR J17511, the line broadness $(\sigma \sim 0.7 \mathrm{keV})$ is intermediate between 170544 and SAX J1808.4. For HETE J1900.1 the pulsations could not be firmly detected, and the spectrum and physical characteristics appear closer to the parameters found for the hard state of 1705-44, but differently from the atoll source, the ionization parameter is between 750 and 1000, while in 4U170544 is $<100$. Moreover, HETE J1900.1 shows more complex reflection features, with a strong excess around $1 \mathrm{keV}$ that is absent in other sources. The reason may be due to a combination of the ionization state and an unusual high metallicity of the system.

The ms X-ray pulsar IGR J17511 showed the highest hard-state luminosity among the sample, and a spectral shape similar to the one of ms pulsar SAX J1808.4. Like HETE J1900.1, the reflecting disk is highly ionized and disk is truncated at a significant distance from the NS surface. In all the accreting ms pulsars, the disk truncation resulted always close to the co-rotational radius.

In conclusion, the presence of pulsations seem not sufficient to foresee the shape of the reflection component. Between non-pulsating and pulsating sources, the inner disk radius is presently known with little accuracy to make it a suitable test for discriminating presence or absence of a magnetic barrier. The ionization state of the disk is also strongly variable and appears uncorrelated with the source luminosity, or with the disk inner radius.

\section{References}

[1] A. D’Aì, R. Iaria, T. Di Salvo, G. Matt, N.R. Robba, ApJ 693, L1 (2009), 0812 . 1974

[2] T. Di Salvo, A. D’Aí, R. Iaria, L. Burderi, M. Dovčiak, V. Karas, G. Matt, A. Papitto, S. Piraino, A. Riggio et al., MNRAS 398, 2022 (2009), 0904 . 3318

[3] R. Iaria, A. D’Aí, T. di Salvo, N.R. Robba, A. Riggio, A. Papitto, L. Burderi, A\&A 505, 1143 (2009), 0906. 3716

[4] S. Piraino, A. Santangelo, P. Kaaret, B. Mück, A. D’Aì, T. Di Salvo, R. Iaria, N. Robba, L. Burderi, E. Egron, A\&A 542, L27 (2012), 1205. 3348

[5] A. D’Aì, T. di Salvo, D. Ballantyne, R. Iaria, N.R. Robba, A. Papitto, A. Riggio, L. Burderi, S. Piraino, A. Santangelo et al., A\&A 516, A36 (2010), 1004. 1963

[6] E. Egron, T. Di Salvo, S. Motta, L. Burderi, A. Papitto, R. Duro, A. D’ Aì, A. Riggio, T. Belloni, R. Iaria et al., A\&A 550, A5 (2013), 1211.2933

[7] A. Papitto, T. Di Salvo, A. D’Aì, R. Iaria, L. Burderi, A. Riggio, M.T. Menna, N.R. Robba, A\&A 493, L39 (2009), 0812 . 1149

[8] A. Papitto, A. Riggio, T. di Salvo, L. Burderi, A. D’Aì, R. Iaria, E. Bozzo, M.T. Menna, MNRAS 407, 2575 (2010), 1005. 4827

[9] A. Papitto, A. D’Aì, T. Di Salvo, E. Egron, E. Bozzo, L. Burderi, R. Iaria, A. Riggio, M.T. Menna, MNRAS 429, 3411 (2013), 1212.2532 